\section{Dr. Abel Monfort}

ESIC Business \& Marketing School

Departamento de Dirección de Empresas

@ abel.monfort@esic.edu (iD 0000-0002-3713-7102

Dr. José Manuel Mas Iglesias

ESIC Business \& Marketing School

Departamento de Dirección de Marketing

@ josemanuel.mas@esic.edu

(iD $0000-0003-2931-1235$
Recibido / Received 8 de noviembre de 2020

- Aceptado / Acepted 16 de noviembre de 2020

- Páginas / Pages De la 349 a la 361

- ISSN: 1885-365X

\title{
Barreras y oportunidades para la comunicación de la responsabilidad social en redes sociales
}

\section{Communicating CSR in social media: challenges and opportunities}

\section{RESUMEN:}

El objetivo del artículo es realizar una revisión de la literatura sobre las barreras y oportunidades de la comunicación en redes sociales de los contenidos vinculados con la Responsabilidad Social Corporativa (RSC) y la gestión de la sostenibilidad. Para ello, se han seleccionado 466 artículos de Web of Science con palabras clave relacionadas con el objetivo. Para el análisis, solo se han tenido en cuenta aquellas publicaciones en revistas científicas y escritas en inglés. El número total de artículos analizados tras este filtro ha sido de 72. Los principales intereses de la RSC en redes sociales son: i) el diálogo de los stakeholders; ii) ganar credibilidad, legitimidad y reputación; iii) mejorar el engagement y iv) el impacto de comunicación de la RSC y la sostenibilidad en el marketing y el marketing social.

\section{PALABRAS CLAVE:}

Comunicación corporativa; Responsabilidad Social Corporativa (RSC); Gestión de la sostenibilidad; Relaciones con los grupos de interés; Redes sociales.

\section{ABSTRACT:}

The aim of the paper is to conduct a literature review on the barriers and opportunities of social media communication of content related to Corporate Social Responsibility (CSR) and sustainability management. To this end, 466 articles from the Web of Science have been selected with keywords related to the objective. Only those publications in scientific journals and written in English have been considered for analysis. The total number of articles analyzed after this filter has been 72. The main interests of CSR in social networks are: i) the dialogue of stakeholders, ii) gaining credibility, legitimacy and reputation, iii) improving engagement and iv) the communication impact of CSR and sustainability in marketing and social marketing.

Corporate Communication; Corporate Social Responsibility (CSR); Sustainability Management; Stakeholder Relations; Social Networks. 


\section{Introducción}

La Responsabilidad Social Corporativa (RSC) tiene como principal objetivo la creación de valor para los grupos de interés (Peloza y Shang, 2011). Mientras tanto, la comunicación corporativa permite a los stakeholders obtener transparencia, información sobre el enfoque comercial de la empresa y el propósito corporativo (Du, Bhattacharya y Sen, 2010; Jahdi y Acikdilli, 2009). En este contexto, las redes sociales han cambiado profundamente el escenario de la comunicación y el desarrollo de la RSC. Con las redes sociales, las empresas pierden el control sobre la marca y la reputación (Macnamara y Zerfass, 2012) ante unas audiencias que interactúan entre sí y con la empresa. Sin embargo, la comunicación digital también ofrece la posibilidad de interactuar con audiencias más grandes (DiStaso, McCorkindale y Wright, 2011) y de fomentar el diálogo. Desde la perspectiva de la RSC, estas herramientas pueden ayudar a las empresas a mejorar su objetivo y su compromiso en términos de sostenibilidad y diálogo.

La literatura sugiere que, a pesar de estos beneficios, las empresas no hacen un buen uso de las redes sociales para fomentar la conversación (Illia et al., 2017), ya que no facilitan el diálogo, el engagement, ni un enfoque multistakeholder a la hora de poner en práctica su estrategia de comunicación (Elving y Postma, 2017). Además, las empresas son conscientes de que las redes sociales atraen la atención del público cuando se producen comportamientos irresponsables (Elving y Postma, 2017). Sin embargo, saben que las redes sociales les permiten compartir las actividades de RSC así como identificar las expectativas de los stakeholders (Eberle, Berens y Li, 2013).

Esta investigación tiene como objetivo analizar y estructurar las principales características de la comunicación de contenidos relacionados con la RSC y la sostenibilidad en las redes sociales. Las preguntas de investigación que abordamos en este estudio son las siguientes: ¿Cuáles son las ventajas de comunicar la RSC de la organización a través de RRSS? ¿Cuáles son las barreras y las dificultades de este medio para transmitir información relativa a la RSC?

Para aportar luz sobre estos problemas, el estudio analiza las principales publicaciones sobre RSC y comunicación en redes sociales publicadas en los últimos años en revistas científicas indexadas en la colección principal de la Web of Science (WoS). Para la realización de la búsqueda en WoS se han tenido en cuenta las siguientes palabras clave: «CSR or Sustainability or Ethics» seguido de «social media or social networks o el nombre específico de una plataforma» (ej. Twitter, Facebook, Instagram, Tiktok...). De los 466 artículos seleccionados, solo aquellos publicados en las revistas científicas y escritos en inglés se han tenido en cuenta para el análisis. El número total de artículos analizados tras este filtro ha sido de 72 .

La mayor parte de los estudios que versan sobre la Responsabilidad Social Corporativa y la sostenibilidad se basan en la importancia de las relaciones entre las empresas y sus grupos de interés. Esta aproximación se fundamenta en la teoría institucional, que expone que las instituciones adoptan normas y se ajustan a la regulación vigente que, unida a los elementos culturales, proporcionan estabilidad e impactan en la sociedad (Scott, 2014, p. 56). El proceso de institucionalización debe basarse en los tres pilares que dan vida y consistencia a dicha teoría. Estos son: la regulación, la normativa y los elementos culturales cognitivos. En consecuencia, las organizaciones no solo tienen que desarrollar los recursos económicos que les permitan alcanzar su actividad y diferenciarse, sino que además deben adoptar conduc- 
tas y estrategias que les permitan situarse dentro de la sociedad, como una institución fiable y respetable.

En otras palabras, tal y como lo describe Suchman (1995, p. 574), las empresas deben actuar dentro de marco normativo, de los valores y las buenas conductas sociales, permitiendo que se sitúen en un marco de aceptación social, y así estar legitimadas frente a sus grupos de interés. Desde este punto de vista de legitimación frente a los grupos de interés (a menudo considerado como una de las bases teóricas de la reputación corporativa), la alta dirección debe ser consciente de que la empresa opera bajo un entorno multistakeholder, entendido como el contexto formato por todas aquellas personas $u$ organizaciones que pueden impactar o ser impactadas por la actividad de la propia empresa (Freeman, 1984). Por todo esto, la gestión de la responsabilidad social y la sostenibilidad en las compañías provoca una nueva forma de entender los modelos de negocio y las estrategias de comunicación.

\section{Sostenibilidad y diálogo}

El estudio de Morsing y Schultz (2006) se caracteriza porque profundizó en la tipología del diálogo y su impacto en la estrategia corporativa y la comunicación en las organizaciones. Estos autores establecieron tres posibles estrategias para establecer un diálogo. Más tarde, Colleoni (2013) expandió estas estrategias y su impacto en las redes sociales. Monfort, Villagra y López-Vázquez (2019) integraron estas estrategias tal y como se observa en la tabla 1 (las diferentes estrategias de diálogo implican una serie de estilos comunicativos):

Sin embargo, hay diferentes estudios que demuestran que, a pesar de la creación de mecanismos corporativos para desarrollar un dialogo a través de las redes sociales con los grupos de interés, muchas veces no consiguen su objetivo (Illia et al., 2017). Por ello, esta investigación persigue descubrir las oportunidades y limitaciones que ofrecen las redes sociales en la comunicación y diálogo de la RSC y la sostenibilidad.

\section{Barreras y oportunidades para la comunicación de la sostenibilidad a través de las redes sociales}

\subsection{Barreras para de la comunicación de la Responsabilidad Social Corporativa}

Los estudios publicados en los últimos años se han enfocado en: i) análisis del diálogo entre la empresa y sus grupos de interés; ii) la consecución de la credibilidad, legitimación y la reputación y iii) la búsqueda de métodos comunicativos que mejoren la conexión con la audiencia, y el compromiso de dicho público.

Los estudios que analizan el diálogo con los grupos de interés alertan de la falta de comunicación real entre empresa y stakeholders. Abitbol, Meeks y Cummins (2019) demostraron que, a pesar de los esfuerzos que realizan las empresas por difundir sus actividades de RSC, acaban utilizando una comunicación unidireccional por la falta de un lenguaje que favorezca 
Tabla 1. Diálogo con los stakeholders en la comunicación de la RSC

\begin{tabular}{|c|c|c|c|}
\hline Estrategia & Escenario & Comunicación & Ejemplos \\
\hline $\begin{array}{l}\text { Estrategia de } \\
\text { Información } \\
\text { (Morsing y } \\
\text { Schultz, 2006) }\end{array}$ & $\begin{array}{l}\text { Las expectativas de } \\
\text { los grupos de interés } \\
\text { no están integradas en } \\
\text { la visión corporativa }\end{array}$ & $\begin{array}{l}\text { Información corporativa } \\
\text { unidireccional }\end{array}$ & $\begin{array}{l}\text { Dosieres de prensa, } \\
\text { catálogos, campañas } \\
\text { publicitarias }\end{array}$ \\
\hline $\begin{array}{l}\text { Estrategia de } \\
\text { Respuesta } \\
\text { (Morsing, Schultz } \\
\text { y Nielsen, 2008; } \\
\text { Morsing y } \\
\text { Schultz, 2006) }\end{array}$ & $\begin{array}{l}\text { Los valores } \\
\text { corporativos son } \\
\text { definidos internamente }\end{array}$ & \multirow{2}{*}{$\begin{array}{l}\text { Los valores se } \\
\text { comunican después } \\
\text { de estudiar el impacto } \\
\text { específico en los grupos } \\
\text { de interés }\end{array}$} & \multirow{3}{*}{$\begin{array}{l}\text { Informes de RSC, } \\
\text { paneles de expertos, } \\
\text { reuniones anuales, } \\
\text { comunicación interna, } \\
\text { cuestionarios, NPS... }\end{array}$} \\
\hline $\begin{array}{l}\text { Estrategia } \\
\text { comunicativa } \\
\text { centrada en uno } \\
\text { mismo (Colleoni, } \\
\text { 2013) }\end{array}$ & \multirow{2}{*}{$\begin{array}{l}\text { La estrategia de } \\
\text { RSC está definida } \\
\text { internamente, } \\
\text { pero teniendo en } \\
\text { cuenta las posibles } \\
\text { reacciones sobre el } \\
\text { posicionamiento }\end{array}$} & & \\
\hline $\begin{array}{l}\text { Estrategia de } \\
\text { mediación } \\
\text { (Colleoni, 2013) }\end{array}$ & & $\begin{array}{l}\text { Los expertos analizan } \\
\text { el posible impacto } \\
\text { y provocan que los } \\
\text { mensajes se emitan } \\
\text { por otros actores. } \\
\text { Conectan la información } \\
\text { corporativa con otros } \\
\text { grupos de interés }\end{array}$ & \\
\hline $\begin{array}{l}\text { Estrategia } \\
\text { participativa } \\
\text { a través del } \\
\text { diálogo (Colleoni, } \\
\text { 2013; Morsing y } \\
\text { Schultz, 2006) }\end{array}$ & $\begin{array}{l}\text { El diálogo entre las } \\
\text { partes da lugar a } \\
\text { la definición de los } \\
\text { valores corporativos y } \\
\text { la estrategia de la RSC }\end{array}$ & $\begin{array}{l}\text { Se crean junto con los } \\
\text { stakeholders valores } \\
\text { y objetivos de interés } \\
\text { común }\end{array}$ & $\begin{array}{l}\text { Redes sociales, } \\
\text { reuniones con grupos } \\
\text { de interés, foros de } \\
\text { debate, cátedras } \\
\text { universitarias, páginas } \\
\text { web participativas... }\end{array}$ \\
\hline
\end{tabular}

Fuente: Adaptación de Monfort, Villagra y López-Vázquez (2019).

la interacción y la conversación. Un estudio llevado a cabo en América Latina refuerza este argumento, pues asegura que el modo de comunicación de la RSC en las redes sociales no es bidireccional, provocando bajo interés por el diálogo entre grupos de interés (Capriotti y Zeler, 2020). Monfort, Villagra y López-Vázquez (2019) también demostraron que la falta de diálogo entre las empresas y los usuarios de estas redes hace imposible una creación conjunta de valores y actividades que fomenten la RSC de las organizaciones. En conclusión, las redes sociales son utilizadas como un medio unidireccional para trasmitir información, (Zeler y Capriotti, 2019). En cualquier caso, este diálogo bidireccional depende también de los grupos de interés y de su poder y capacidad de influencia (Saxton, Ren y Guo, 2020).

Respecto a la legitimidad, credibilidad y reputación, la literatura demuestra que el contenido generado por los usuarios es más creíble y otorga más autenticidad que el que tiene ori- 
gen en la compañía (Dunn y Harness, 2019). También, los mensajes cara a cara emitidos por los propios trabajadores (en especial sobre temas controvertidos de la organización) tienen un mayor efecto en la audiencia que los mensajes positivos difundidos por los trabajadores a través de las redes sociales (Lee \& Tao, 2020). El contenido generado por el usuario y la capacidad de recomendar cara a cara también repercute en la reputación.

Recientemente, se ha demostrado que la importancia y el tono de las noticias de RSE en los medios de comunicación tradicionales se relacionan positivamente con la reputación de una empresa, incluso si la noticia es negativa. Sin embargo, en redes sociales, no se ha demostrado la existencia de un vínculo claro entre las noticias de RSE de la empresa y su reputación (Vogler y Eisenegger, 2020). Por lo tanto, en la literatura previa se ha observado que el contenido generado por el usuario, las comunicaciones cara a cara y el poder de los medios tradicionales siguen siendo fuentes clave de reputación. Por último, la preocupación por el uso de datos personales por parte de las empresas hace que los stakeholders perciban un mayor riesgo cuando comparten información en internet, lo que conduce a opiniones falsas (Michaelidou y Micevski, 2019).

En lo que respecta al compromiso y la comunicación en materia de RSC, se ha observado que no hay una diferenciación clara en los mensajes de RSC de las empresas, lo que provoca una serie de mensajes que parecen imitar las estrategias de unos y otros (PaliwodaMatiolanska, Smolak-Lozano y Nakayama, 2020). En consecuencia, las empresas deberían contar con cuentas específicas por grupo de interés y crear contenidos adecuados para los usuarios que las siguen (Okazaki et al., 2020). Algunos estudios sobre la mejora del engagement en YouTube han observado que, cuando se utiliza una estrategia muy informativa y orientada a negocio, los comentarios son más bien negativos y escépticos (Song y Wen, 2020). Por ejemplo, en la industria del aceite de palma se ha observado que los fabricantes europeos se limitan a responder a los usuarios, simplemente proporcionando información y evitando la participación en cuestiones delicadas (Ruggeri y Samoggia, 2018). Asimismo, los pocos estudios que han hecho comparaciones internacionales también insisten en la escasa diferenciación de los mensajes en las marcas globales. En cualquier caso, estos estudios han demostrado que la cultura afecta a la forma en que se reacciona a los mensajes (por ejemplo, no es lo mismo un estilo individualista que uno más colectivista) (Ngai, Einwiller y Singh, 2020) (Tabla 2).

Algunos estudios han demostrado que el aumento del compromiso con la audiencia en cuestiones de responsabilidad social de las empresas depende de un mínimo de resonancia previa del mensaje. Es decir, cuanto mayor sea el número de interacciones previas con el mensaje (likes, comentarios, shares...) mayor será la probabilidad de que los usuarios se sientan atraídos a compartir o participar en ese mensaje. Por lo tanto, las empresas deben lograr un mínimo de engagement para aumentar la interacción (Hartmann et al., 2020). Además de una mínima interacción, otra barrera que se ha demostrado en la literatura reciente es el verdadero poder de las celebrities o influencers. Por ejemplo, McKeown y Shearer (2019) analizaron la reacción de los usuarios ante la moda sostenible promovida por la actriz Emma Watson en una red social y determinaron que la existencia de estas influencers no tiene un impacto significativo en la compra de este tipo de moda. 
Tabla 2. Barreras de la comunicación de la RSC de las empresas.

\begin{tabular}{|c|c|c|}
\hline Objetivo & Barrera & Autor/es \\
\hline \multirow{4}{*}{$\begin{array}{l}\text { Diálogo entre } \\
\text { la empresa y } \\
\text { stakeholders }\end{array}$} & $\begin{array}{l}\text { No existe una comunicación bidireccional real ni un } \\
\text { lenguaje interactivo para entablar un diálogo con los } \\
\text { stakeholder }\end{array}$ & $\begin{array}{l}\text { (Abitbol, Meeks y } \\
\text { Cummins, 2019; } \\
\text { Capriotti y Zeler, 2020) }\end{array}$ \\
\hline & $\begin{array}{l}\text { Prevalece un estilo informativo con una falta de interés } \\
\text { por el diálogo }\end{array}$ & (Capriotti y Zeler, 2020) \\
\hline & $\begin{array}{l}\text { No hay una conversación real que permita la co- } \\
\text { creación y la comunicación entre la organización y la } \\
\text { audiencia }\end{array}$ & $\begin{array}{l}\text { (Monfort, Villagra y } \\
\text { López-Vázquez, 2019) }\end{array}$ \\
\hline & $\begin{array}{l}\text { El interés de la empresa por dialogar con los usuarios } \\
\text { depende del poder y capacidad de influencia de los } \\
\text { stakeholders }\end{array}$ & (Saxton, Ren y Guo, 2020) \\
\hline \multirow{4}{*}{$\begin{array}{l}\text { Ganar } \\
\text { credibilidad, } \\
\text { legitimidad y } \\
\text { reputación }\end{array}$} & $\begin{array}{l}\text { El contenido generado por el usuario es más creíble y } \\
\text { proporciona más legitimidad que las comunicaciones } \\
\text { corporativas }\end{array}$ & (Dunn y Harness, 2019) \\
\hline & $\begin{array}{l}\text { La credibilidad de los mensajes positivos se ve } \\
\text { socavada cuando un empleado los comparte a través } \\
\text { de las redes sociales o en persona }\end{array}$ & (Lee y Tao, 2020) \\
\hline & $\begin{array}{l}\text { No hay un vínculo claro entre la difusión online de la } \\
\text { actividad de RSE de la empresa y su reputación }\end{array}$ & $\begin{array}{l}\text { (Vogler y Eisenegger, } \\
\text { 2020) }\end{array}$ \\
\hline & $\begin{array}{l}\text { La preocupación por el uso de datos digitales por } \\
\text { parte de las empresas hace que los stakeholders } \\
\text { perciban un mayor riesgo al compartir información, lo } \\
\text { que da lugar a opiniones falsas }\end{array}$ & $\begin{array}{l}\text { (Michaelidou y Micevski, } \\
\text { 2019) }\end{array}$ \\
\hline \multirow{6}{*}{$\begin{array}{l}\text { Aumento de } \\
\text { engagement }\end{array}$} & $\begin{array}{l}\text { Hay una falta de diferenciación en el contenido de } \\
\text { la RSE de las empresas, lo que lleva a una imitación } \\
\text { mutua de las estrategias de diálogo }\end{array}$ & $\begin{array}{l}\text { (Paliwoda-Matiolanska, } \\
\text { Smolak-Lozano y } \\
\text { Nakayama, 2020) }\end{array}$ \\
\hline & $\begin{array}{l}\text { Las empresas deben tener en cuenta a usuarios } \\
\text { específicos y crear un contenido apropiado para el } \\
\text { público que les sigue }\end{array}$ & (Okazaki et al., 2020) \\
\hline & $\begin{array}{l}\text { Los comentarios son más negativos y escépticos } \\
\text { cuando las empresas utilizan una estrategia muy } \\
\text { informativa y orientada a los negocios }\end{array}$ & (Song y Wen, 2020) \\
\hline & $\begin{array}{l}\text { La cultura afecta a la forma en que se reacciona a los } \\
\text { mensajes }\end{array}$ & $\begin{array}{l}\text { (Ngai, Einwiller y Singh, } \\
\text { 2020) }\end{array}$ \\
\hline & $\begin{array}{l}\text { Las empresas deben lograr un mínimo de compromiso } \\
\text { para aumentar la resonancia de los mensajes }\end{array}$ & (Hartmann et al., 2020) \\
\hline & $\begin{array}{l}\text { Influencers y celebrities no tienen un impacto } \\
\text { significativo en la intención de compra }\end{array}$ & $\begin{array}{l}\text { (McKeown y Shearer, } \\
\text { 2019) }\end{array}$ \\
\hline
\end{tabular}




\subsection{Oportunidades para la comunicación de la Responsabilidad Social Corporativa}

Algunos estudios publicados en los últimos años arrojan resultados positivos para las oportunidades de las estrategias de comunicación de la responsabilidad social corporativa. Estos estudios están principalmente enfocados en: i) cómo mejorar el diálogo entre la empresa y sus grupos de interés; ii) el aumento de la credibilidad, legitimación y la reputación; iii) la búsqueda de métodos comunicativos que mejoren la conexión con la audiencia, y el compromiso de dicho público y iv) el impacto de estos métodos de comunicación en las estrategias de marketing.

Sobre el diálogo, la mera participación en redes sociales puede ayudar a establecer una conversación con los grupos de interés que mejore la responsabilidad social corporativa (Barbeito-Caamaño y Chalmeta, 2020), a través del uso de diferentes cuentas en redes sociales, identificando cada stakeholder (Huang et al., 2019; Zhang, Gosselt y De Jong, 2020)

Las oportunidades para mejorar la credibilidad, legitimación y reputación se centran en el estudio del contenido usado en las estrategias de comunicación en redes sociales, resaltando la importancia de este. La consecución de estos atributos juega un papel fundamental en la forma en la que las empresas reducen el escepticismo y aumentan el compromiso de la marca (Dunn y Harness, 2018). Aquí los estudios muestran cómo la presencia de marca en redes sociales mejora el posicionamiento y evaluación de su responsabilidad social corporativa, frente a competidores similares, aunque también aumenta la presión sobre la marca debido a su alta exposición. Dicho de otra forma, tener presencia en Twitter obliga a las empresas a tener una mejor política de responsabilidad social corporativa que competidores similares, y de esta forma controlar las expectativas de transparencia y responsabilidad (Balasubramanian, Fang y Yang, 2020).

En términos de oportunidades para mejorar la conexión con la audiencia a través del contenido publicado sobre RSC, los estudios muestran cómo los mensajes de estilo aspiracional, así como el aumento de la frecuencia de estos mensajes, provocan un incremento del alcance y recomendación por parte de los usuarios (Araujo y Kollat, 2018). De igual forma, los usuarios muestran preferencias por formatos como infografías (Huang et al., 2019). En YouTube, las compañías deben permitir siempre los comentarios sobre los videos publicados con material de RSC y así, favorecer la interacción de los individuos frente a las políticas de sostenibilidad de la empresa (Liao y Mak, 2019). Se debe tener en cuenta, además, la temática de la RSC de cara a evaluar su capacidad de conexión con la audiencia. Se ha estudiado, por ejemplo, cómo en el sector financiero, el contenido relativo a RSC en el campo de la ética o del ámbito legal, tiene mayor importancia percibida que lo relativo a la filantropía (Wang y Pala, 2020).

Otros estudios sobre el sector asegurador han demostrado cómo los mensajes en Twitter con contenido de RSC relativos al negocio, resultados financieros y actividades llevadas a cabo en las comunidades donde la compañía opera, aumentan el engagement en las redes sociales (Monfort, Villagra y López-Vázquez, 2019). Saxton et al. (2019) analizaron las cuentas de Twitter del listado Fortune 500 y concluyeron que existe mayor interacción (retweets) a través de contenido con temática RSC relativo a educación o medio ambiente, cuando se utilizan hashtags o cuando las compañías se unen al diálogo o discusión sobre un movimiento social preexistente. En resumen, el sector desempeña un papel fundamental en la búsqueda de engagement a través de la comunicación de contenidos relacionados con RSC. 
Un ámbito de estudio poco trabajado en la búsqueda del engagement a través del contenido de RSC es el relativa a la narrativa transmedia, transmedia storytelling en inglés. A través del estudio de una campaña de $P \& G$, se pudo observar que la narrativa transmedia ayuda a que los mensajes de RSC provoquen reacciones emocionales positivas, evitando así otras reacciones negativas provocadas por otras formas de comunicar RSC como pudiera ser la publicidad tradicional (Coombs, 2019).

Otra área que muestra oportunidades para las estrategias de comunicación de RSC en redes sociales es el ámbito del marketing y el marketing social. A través de una encuesta a usuarios de redes sociales en China se demostró que las conversaciones y otras actividades relativas a la RSC de una compañía, impactaban en la intención de compra debido a un aumento de la identificación de la marca (Chu y Chen, 2019). Por ello, cuando un mensaje de RSC es percibido como relevante, éste mejora la credibilidad de la marca, así como la intención de compra y el eWOM en redes sociales (Hazel y Kang, 2018). Tras analizar el comportamiento en redes sociales de los milenials estadounidenses frente a los productos sostenibles, se determinó que el uso de las redes sociales y la influencia social tienen un efecto positivo en la intención de compra frente a estos productos (Bedard y Tolmie, 2018).

Finalmente, el apoyo de celebridades e influencers es determinante para la efectividad de las campañas de marketing social (Holiday et al., 2020), así como para promover la concienciación (McKeown y Shearer, 2019). Para tal fin, el uso de diferentes canales es apropiado, junto con los medios sociales, así como la repetición de los mensajes a lo largo del tiempo (Young et al., 2018) (Tabla 3).

\section{Conclusiones/Discusión}

Este estudio analiza las principales barreras y oportunidades para la RSC y la sostenibilidad en las redes sociales. La revisión de la literatura ha demostrado que los principales intereses de la comunicación de RSC en redes sociales son: i) el diálogo de los stakeholders, ii) ganar credibilidad, legitimidad y reputación, iii) mejorar el engagement y iv) el impacto de la comunicación de la RSC y la sostenibilidad en el marketing y en el marketing social.

En cuanto al diálogo, la situación se ha mantenido igual desde hace años. Una de las principales barreras para la comunicación es que el enfoque sigue siendo unidireccional. Las empresas están operando bajo los principios de la estrategia de la información (Morsing y Schultz, 2006), creándose perfiles en redes sociales pero siguen sin interactuar realmente con la audiencia (Illia et al., 2017). Sería interesante desarrollar nuevas investigaciones que dejasen de lado el estudio del comportamiento en redes y se centrasen en analizar a las personas que toman las decisiones para saber exactamente el motivo de la falta de interés en la conversación y el diálogo. Por otro lado, dado que parece claro que no hay interés en el diálogo por parte de las empresas, sería conveniente conocer qué temas interesan a los stakeholders. Analizando ambos discursos, sería posible entender exactamente la magnitud de esta falta de interés en el diálogo e intentar abordarla. A pesar de todas estas dificultades, la presencia en redes sociales es ya un paso hacia adelante (Barbeito-Caamaño y Chalmeta, 2020).

En cuanto a la legitimidad, los estudios muestran que la mera presencia en las redes sociales aumenta la legitimidad porque hace que las empresas mejoren sus políticas de RSC 
Table 3. Oportunidades para la comunicación de la responsabilidad social corporativa

\begin{tabular}{|c|c|c|}
\hline Objetivo & Oportunidad & Autor/s \\
\hline \multirow{2}{*}{$\begin{array}{l}\text { Diálogo entre } \\
\text { empresa y grupos } \\
\text { de interés }\end{array}$} & $\begin{array}{l}\text { La participación en redes sociales ayuda a } \\
\text { mejorar la RSC y el diálogo con los grupos de } \\
\text { interés }\end{array}$ & $\begin{array}{l}\text { (Barbeito-Caamaño } \\
\text { y Chalmeta, 2020) }\end{array}$ \\
\hline & $\begin{array}{l}\text { El uso de diferentes cuentas en redes } \\
\text { sociales, identificando cada grupo de interés, } \\
\text { favorece la comunicación con los mismos }\end{array}$ & $\begin{array}{l}\text { (Huang et al., 2019; Zhang, } \\
\text { Gosselt y De Jong, 2020) }\end{array}$ \\
\hline \multirow[b]{2}{*}{$\begin{array}{l}\text { Ganar credibilidad, } \\
\text { legitimidad y } \\
\text { reputación }\end{array}$} & $\begin{array}{l}\text { El contenido en redes sociales puede mejorar } \\
\text { la credibilidad, legitimación y reputación de la } \\
\text { empresa }\end{array}$ & (Dunn y Harness, 2018) \\
\hline & $\begin{array}{l}\text { Tener redes sociales obliga a las empresas } \\
\text { a desarrollar una mejor política de } \\
\text { responsabilidad social corporativa que los } \\
\text { competidores similares, y de esta forma } \\
\text { controlar las expectativas de transparencia y } \\
\text { responsabilidad }\end{array}$ & $\begin{array}{l}\text { (Balasubramanian, Fang } \\
\text { y Yang, 2020) }\end{array}$ \\
\hline \multirow{5}{*}{$\begin{array}{l}\text { Aumento de } \\
\text { engagement }\end{array}$} & $\begin{array}{l}\text { El carácter aspiracional del mensaje, así } \\
\text { como la frecuencia en las publicaciones de } \\
\text { RSC, aumentan el alcance y recomendación } \\
\text { de los usuarios }\end{array}$ & (Araujo y Kollat, 2018) \\
\hline & $\begin{array}{l}\text { Los usuarios prefieren formatos atractivos } \\
\text { como las infografías }\end{array}$ & (Huang et al., 2019) \\
\hline & $\begin{array}{l}\text { Posibilitar comentarios mejora la } \\
\text { comunicación y la actitud de los individuos } \\
\text { hacía las políticas de sostenibilidad de la } \\
\text { empresa }\end{array}$ & (Liao y Mak, 2019) \\
\hline & $\begin{array}{l}\text { El sector de actividad en el que opera la } \\
\text { compañía influye en el interés generado por el } \\
\text { contenido de RSC comunicado }\end{array}$ & $\begin{array}{l}\text { (Monfort, Villagra } \\
\text { y López-Vázquez, 2019; } \\
\text { Saxton et al., 2019; } \\
\text { Wang y Pala, 2020) }\end{array}$ \\
\hline & $\begin{array}{l}\text { La narrativa transmedia ayuda a provocar } \\
\text { reacciones emocionales positivas bloqueando } \\
\text { otras reacciones negativas causadas por } \\
\text { otras formas de comunicación de la RSC }\end{array}$ & (Coombs, 2019) \\
\hline \multirow{3}{*}{$\begin{array}{l}\text { Impacto en } \\
\text { marketing y } \\
\text { marketing social }\end{array}$} & $\begin{array}{l}\text { Las conversaciones de usuarios sobre las } \\
\text { actividades de RSC impactan positivamente } \\
\text { en la intención de compra debido al aumento } \\
\text { de identificación con la marca }\end{array}$ & (Chu y Chen, 2019) \\
\hline & $\begin{array}{l}\text { Los mensajes de RSC percibidos como } \\
\text { relevantes tienen un impacto positivo en la } \\
\text { confianza de marca, intención de compra y } \\
\text { eWOM }\end{array}$ & $\begin{array}{l}\text { (Bedard y Tolmie, 2018; } \\
\text { Hazel y Kang, 2018) }\end{array}$ \\
\hline & $\begin{array}{l}\text { Celebridades e influencers son esenciales } \\
\text { para promover el reconocimiento de marca }\end{array}$ & $\begin{array}{l}\text { (Holiday et al., 2020; } \\
\text { McKeown y Shearer, 2019) }\end{array}$ \\
\hline
\end{tabular}


y las obliga a mejorar frente a la competencia (Balasubramanian, Fang y Yang, 2020). Sin embargo, hay una serie de barreras que implican muchas preguntas. Se sabe que, en comparación con el discurso de la empresa, el discurso generado por el usuario es más creíble (Dunn y Harness, 2019). El problema es que todavía hay una falta de conocimiento sobre cómo conseguir que los usuarios hablen bien de la empresa y de su política de RSC.

En cuanto a la forma de lograr el engagement en la comunicación sobre la RSC, esta revisión de la literatura ha presentado muchas oportunidades para los profesionales encargados de la gestión de las redes sociales (no a nivel estratégico, sino a nivel operacional). Por ejemplo, demostrando que la literatura recomienda la creación de canales de comunicación específicos para los stakeholders (Huang et al, 2019; Okazaki et al., 2020; Zhang, Gosselt y De Jong, 2020); evitando los post informativos y buscando mensajes aspiracionales, así como publicando frecuentemente noticias sobre RSC (Araujo y Kollat, 2018); siendo sensibles a las diferentes culturas en las que opera la empresa (Ngai, Einwiller y Singh, 2020) y a la industria y la competencia (Monfort, Villagra y López-Vázquez, 2019; Saxton et al., 2019; Wang y Pala, 2020); buscando emociones a través de nuevos formatos de comunicación como la narrativa transmedia en los medios de comunicación (Coombs, 2019); permitiendo comentarios en todos los post para que la empresa pueda hablar con la audiencia (Liao y Mak, 2019) y utilizando formatos más atractivos según las posibilidades de la red (Huang et al., 2019). Así pues, esta línea de investigación está muy desarrollada y ofrece las mayores oportunidades a las empresas. El problema es que se presenta de una manera muy operativa y no estratégica.

Una de las grandes barreras detectadas en la búsqueda del engagement es que hay una clara falta de diferenciación en los mensajes (Paliwoda-Matiolanska, Smolak-Lozano y Nakayama, 2020). En otras palabras, todas las empresas comunican su política de RSE o de sostenibilidad de manera similar. Se necesitan estudios que demuestren si las empresas son capaces de comunicar su propósito de una forma diferenciadora y atractiva.

En cuanto al marketing, las investigaciones han demostrado que la responsabilidad social de las empresas es una fuente de mejora de reputación de la marca (Holiday et al., 2020; McKeown y Shearer, 2019) y que es una oportunidad para las empresas. Sin embargo, sigue sin estar claro cómo aumentar la intención de compra mediante la comunicación de la RSC. Los estudios futuros deberían ahondar en estas características, ya que la literatura sobre este tema es escasa (Bedard y Tolmie, 2018; Chu y Chen, 2019; Hazel y Kang, 2018). El artículo ha demostrado que todavía hay muchas lagunas que cubrir en la investigación sobre la comunicación en línea de la RSE.

\section{Bibliografía}

ABITBOL, Alan, MEEKS, Judson y CUMMINS, Glenn (2019). «Does oil and goodwill mix?: Examining the oil and gas industry's impact on stakeholder engagement on Facebook». Environmental Communication, 13(2), 192-208. DOI: 10.1080/17524032.2018.1546751

ARAUJO, Theo y KOLLAT, Jana (2018). «Communicating effectively about CSR on Twitter: The power of engaging strategies and storytelling elements». Internet Research, 28(2), 419-431. DOI: 10.1108/ IntR-04-2017-0172 
BALASUBRAMANIAN, Siva K., FANG, Yiwei y YANG, Zihao (2020). «Twitter presence and experience improve corporate social responsibility outcomes». Journal of Business Ethics. DOI: 10.1007/ s10551-020-04537-x

BARBEITO-CAAMAÑO, Adriana y CHALMETA, Ricardo (2020). «Using big data to evaluate corporate social responsibility and sustainable development practices». Corporate Social Responsibility and Environmental Management, 27(6), 2831-2848. DOI: 10.1002/csr.2006

BEDARD, Stephanie A. N., TOLMIE, Carri R. (2018). «Millennials' green consumption behaviour: Exploring the role of social media». Corporate Social Responsibility and Environmental Management, 25(6), 1388-1396. DOI: 10.1002/csr.1654

CAPRIOTTI, Paul y ZELER, lleana (2020). «Disseminating Latin American companies' corporate social responsibility on Facebook: A comparative study with global companies». Palabra Clave, 23(2). DOI: 10.5294/pacla.2020.23.2.7

CHU, Shu-Chuan y CHEN, Hsuan-Ting (2019). «Impact of consumers' corporate social responsibilityrelated activities in social media on brand attitude, electronic word-of-mouth intention, and purchase intention: A study of Chinese consumer behavior». Journal of Consumer Behaviour, 18(6), 453462. DOI: $10.1002 / \mathrm{cb} .1784$

COLLEONI, Elanor (2013). "CSR communication strategies for organizational legitimacy in social media». Corporate Communications: An International Journal, 18(2), 228-248. DOI: 10.1108/13563281311319508

COOMBS, Timothy (2019). «Transmedia storytelling: a potentially vital resource for CSR communication». Corporate Communications, 24(2), 351-367. DOI: 10.1108/CCIJ-11-2017-0114

DISTASSO, Marcia W., MCCORKINDALE, Tina y WRIGHT, Donald K. (2011). «How public relations executives perceive and measure the impact of social media in their organizations». Public Relations Review, 37(3), 325-328. DOI: 10.1016/j.pubrev.2011.06.005

DU, Shuili, BHATTACHARYA, C. B. y SEN, Sankar (2010). «Maximizing business returns to corporate social responsibility (CSR): The role of CSR communication». International Journal of Management Reviews, 12(1), 8-19. DOI: 10.1111/j.1468-2370.2009.00276.x

DUNN, Katherine, HARNES, David (2018). «Communicating corporate social responsibility in a social world: the effects of company-generated and user-generated social media content on CSR attributions and scepticism». Journal of Marketing Management, 34(17-18), 1503-1529. DOI: 10.1080/0267257X.2018.1536675

DUNN, Katherine, HARNES, David (2019). «Whose voice is heard? The influence of user-generated versus company-generated content on consumer scepticism towards CSR». Journal of Marketing Management, 35(9-10), 886-915. DOI: 10.1080/0267257X.2019.1605401

EBERLE, David, BERENS, Guido y LI, Ting (2013). "The impact of interactive corporate social responsibility communication on corporate reputation». Journal of Business Ethics, 118(4), 731746. DOI: 10.1007/s10551-013-1957-y

ELVING, Wim J. L., POSTMA, Rosa-May (2017). «Social media: The dialogue myth? How organizations use social media for stakeholder dialogue». In Van Ruler, Betteke, Smit, Lekje, Ihlen, Øyvind y Romenti, Stefania (Eds.), How strategic communication shapes value and innovation in society. Emerald Publishing Limited, págs. 123-141.

FREEMAN, R. Edward (1984). Strategic management. A stakeholder approach (Issue Book, Whole). Pitman Ballinger. 
HARTMANN, Patrick, FERNÁNDEZ, Paula, APAOLAZA, Vanessa, EISEND, Martin y D'SOUZA, Clare (2020). «Explaining viral CSR message propagation in social media: The role of normative influences». Journal of Business Ethics. DOI: 10.1007/s10551-020-04540-2

HAZEL, Desiree y KANG, Jiyun (2018). "The contributions of perceived CSR information substantiality toward consumers' cognitive, affective, and conative responses: The hierarchy of effects model approach». Clothing and textiles research journal, 36(2), 62-77. DOI: 10.1177/0887302X17750747

HOLIDAY, Steven, HAYES, Jameson L., BRITT, Brian, C y LYU, Yuanweei (2020). «The cause effect: the impact of corporate social responsibility advertising on cause consumer engagement behavior after brand affiliation ceases». International JournalofAdvertising. DOI: 10.1080/02650487.2020.1769408

HUANG, Lei, CLARKE, Amelia, HELDSINGER, Natalie y TIAN, Wen (2019). «The communication role of social media in social marketing: a study of the community sustainability knowledge dissemination on Linkedln and Twitter». Journal of Marketing Analytics, 7(2), 64-75. DOI: 10.1057/s41270-01900053-8

ILLIA, Laura, ROMENTI, Stefania, RODRÍGUEZ-CÁNOVAS, Belén, MURTARELLI, Grazia y CARROLL, Craig E. (2017). «Exploring corporations' dialogue about CSR in the digital era». Journal of Business Ethics, 146(1), 39-58. DOI: 10.1007/s10551-015-2924-6

JAHDI, Khosro S. y ACIKDILLI, Gaye (2009). «Marketing communications and corporate social responsibility (CSR): Marriage of convenience or shotgun wedding?». Journal of Business Ethics, 88(1), 103-113. DOI: 10.1007/s10551-009-0113-1

LEE, Yeunjae y TAO, Weiting (2020). «Employees as information influencers of organization's CSR practices: The impacts of employee words on public perceptions of CSR». Public Relations Review, 46(1). DOI: 10.1016/j.pubrev.2020.101887

LIAO, Meng-Qi y MAK, Angela K. Y. (2019). "“Comments are disabled for this video”: A technological affordances approach to understanding source credibility assessment of CSR information on YouTube». Public Relations Review, 45(5). DOI: 10.1016/j.pubrev.2019.101840

MACNAMARA, Jim y ZERFASS, Ansgar (2012). «Social media communication in organizations: The challenges of balancing openness, strategy, and management». International Journal of Strategic Communication, 6(4), 287-308. DOI: 10.1080/1553118X.2012.711402

MCKEOWN, Carolyn y SHEARER, Linda (2019). «Taking sustainable fashion mainstream: Social media and the institutional celebrity entrepreneur». Journal of Consumer Behaviour, 18(5), 406-414. DOI: $10.1002 / \mathrm{cb} .1780$

MICHAELIDOU, Nina, y MICEVSKI, Milena (2019). «Consumers' ethical perceptions of social media analytics practices: Risks, benefits and potential outcomes». Journal of Business Research, 104, 576-586. DOI: 10.1016/j.jbusres.2018.12.008

MONFORT, Abel, VILLAGRA, Nuria y LÓPEZ-VÁZQUEZ, Belén (2019). «Exploring stakeholders' dialogue and corporate social responsibility (CSR) on Twitter». Profesional de la informacion, 28(5). DOI: 10.3145/epi.2019.sep.13

MORSING, Mette y SCHULTZ, Majken (2006). «Corporate social responsibility communication: stakeholder information, response and involvement strategies". Business Ethics: A European Review, 15(4), 323-338. DOI: 10.1111/j.1467-8608.2006.00460.x

MORSING, Mette, SCHULTZ, Majken y NIELSEN, Kasper U. (2008). «The 'Catch 22' of communicating CSR: Findings from a Danish study». Journal of Marketing Communications, 14(2), 97-111. DOI: 10.1080/13527260701856608 
NGAI, Cindy S. B., EINWILLER, Sabine y SINGH, Rita G. (2020). «An exploratory study on content and style as driving factors facilitating dialogic communication between corporations and publics on social media in China». Public Relations Review, 46(1). DOI: 10.1016/j.pubrev.2019.101813

OKAZAKI, Shintaro, PLANGGER, Kirk, WEST, Douglas y MENÉNDEZ, Héctor D. (2020). «Exploring digital corporate social responsibility communications on Twitter». Journal of Business Research, 117, 675-682. DOI: 10.1016/j.jbusres.2019.09.006

PALIWODA-MATIOLANSKA, Adriana, SMOLAK-LOZANO, Emilia y NAKAYAMA, Atsuho (2020). «Corporate image or social engagement: Twitter discourse on corporate social responsibility (CSR) in public relations strategies in the energy sector». Profesional de la informacion, 29(3), 1-16. DOI: 10.3145/epi.2020.may.33

PELOZA, John y SHANG, Jingzhi (2011). «How can corporate social responsibility activities create value for stakeholders? A systematic review». Journal of the Academy of Marketing Science, 39(1), 117135. DOI: $10.1007 / \mathrm{s} 11747-010-0213-6$

RUGGERI, Arianna y SAMOGGIA, Antonella (2018). "Twitter communication of agri-food chain actors on palm oil environmental, socio-economic, and health sustainability". Journal of Consumer Behaviour, 17(1), 75-93. DOI: 10.1002/cb.1699

SAXTON, Gregory D., GOMEZ, Lina, NGOH, Zed, LIN, Yi-Pin y DIETRICH, Sarah (2019). «Do CSR messages resonate? Examining public reactions to firms' CSR efforts on social media». Journal of Business Ethics, 155(2), 359-377. DOI: 10.1007/s10551-017-3464-z

SAXTON, Gregory D., REN, Charlotte y GUO, Chao (2020). «Responding to diffused stakeholders on social media: Connective power and firm reactions to CSR-related Twitter messages». Journal of Business Ethics. DOI: 10.1007/s10551-020-04472-x

SCOTT, W. Richard (2014). Institutions and organizations: Ideas and interests. Sage.

SONG, Baobao y Wen, Jing (2020). "Online corporate social responsibility communication strategies and stakeholder engagements: A comparison of controversial versus noncontroversial industries». Corporate Social Responsibility and Environmental Management, 27(2), 881-896. DOI: 10.1002/ csr.1852

SUCHMAN, Mark C. (1995). «Managing legitimacy: Strategic and institutional approaches». Academy of Management Review, 20(3), 571-610. DOI: 10.2307/258788

VOGLER, Daniel y EISENEGGER, Mark (2020). "CSR communication, corporate reputation, and the role of the news media as an agenda-setter in the digital age. Business and Society. DOI: 10.1177/0007650320928969

WANG, Yijing y PALA, BUKET (2020). «Communicating philanthropic CSR versus ethical and legal CSR to employees: empirical evidence in Turkey». Corporate Communications. DOI: 10.1108/CCIJ-012020-0014

YOUNG, C. William, RUSSELL, Sally V., ROBINSON, Cheryl A. y CHINTAKAYALA, Phani K. (2018). "Sustainable retailing - Influencing consumer behaviour on food waste». Business Strategy and the Environment, 27(1), 1-15. DOI: 10.1002/bse.1966

ZELER, lleana y CAPRIOTTI, Paul (2019). «Communicating corporate social responsibility issues on Facebook's corporate fanpages of Latin American companies». Profesional de la informacion, 28(5). DOI: 10.3145/epi.2019.sep.07

ZHANG, Shu, GOSSELT, Jordy F. y DE JONG, Menno D. T. (2020). «How large information technology companies use Twitter: Arrangement of corporate accounts and characteristics of Tweets". Journal of Business and Technical Communication, 34(4), 364-392. DOI: 10.1177/1050651920932191 